\title{
Martín Rodríguez Vivanco y la Sociología de la educación en Cuba
}

\author{
Lídice Mesa Gómez ${ }^{1 \mathrm{i}}$ \\ ${ }^{1}$ Universidad de Artemisa - UA. Asesora do Rector de la Universidad de Artemisa. Rúa 8C, 713, Provincia \\ Artemisa. Cuba. lidicemg@uart.edu.cu.
}

\begin{abstract}
RESUMEN. El presente artículo, tiene el objetivo de revelar la contribución de Martín Rodríguez Vivanco al desarrollo de la sociología de la educación en Cuba durante la República neocolonial y la trascendencia de sus concepciones hasta la actualidad. Se realizó una profunda consulta bibliográfica y como resultado se constató la asunción en su obra de adelantos didácticos del movimiento de la Escuela Nueva, los que adecuó creadoramente al contenido de la asignatura Sociología Pedagógica que impartía en la Universidad de La Habana. La sistematización realizada, reveló que sus concepciones acerca de la teoría de la sociología de la educación se expresan en el tratamiento que hizo de su objeto de estudio, su relación con otras ciencias, sus categorías principales, entre otros, que constituyen el punto de partida para el estudio de esta ciencia en el país y sirven para esclarecer y sustentar las raíces de la pedagogía cubana.
\end{abstract}

Palabras clave: Martín Rodríguez Vivanco, Sociología Pedagógica, Sociología de la Educación. 


\title{
Martín Rodríguez Vivanco and the Sociology of the education in Cuba
}

\begin{abstract}
The present article, has the objective to expose about the contribution of Martín Rodríguez Vivanco related to the sociology development of the education in Cuba during the neo-colonial Republic period and the transcendence of its conceptions at the present time. A deep bibliographic consultation was done and as a result was verified the assumption in his work of didactic advances of the motion of the New School, the ones that made suitable creatively to the content of the subject Pedagogic Sociology that he used to teach at the University of Havana. The realized systematization, showed that Martin Rodriguez Vivanco conceptions about the sociology of the education theory are expressed at the treatment that he made about its object of study, the relation with other sciences, its main categories, among others, that constitute the point of departure to the study of this science in the country and serve to clear and support the roots of the cuban pedagogy.
\end{abstract}

Key words: Martín Rodríguez Vivanco, Pedagogic Sociology, Sociology of the Education. 


\section{Martin Rodriguez Vivanco e sociologia da educação em Cuba}

RESUMO. Este artigo tem como objetivo revelar a contribuição do desenvolvimento Martin Rodriguez Vivanco da sociologia da educação em Cuba durante a República neocolonial e o significado de seus conceitos para hoje. Foi realizada uma busca minuciosa da literatura e como resultado a suposição foi encontrado em seu trabalho de ensino avança o movimento da Escola Nova, que criativamente adaptados ao conteúdo do curso Pedagógico Sociologia que ensinou na Universidade de Havana. Sistematização realizada revelou seus pontos de vista sobre a teoria da sociologia da educação, refletida no tratamento que fez seu assunto, sua relação com outras ciências, suas principais categorias, entre outros, que são o ponto de partida para o estudo desta ciência no país e servir para esclarecer e apoiar as raízes da pedagogia cubana.

Palavras-chave: Martin Rodriguez Vivanco, Sociologia Pedagógica, Sociologia da Educação. 


\section{Introducción}

El progreso social impone a la educación importantes retos, tal es el caso de la conceptualización del aparato teórico que sustenta ese gran edificio que es la educación nacional de cada país. En ese sentido, Cuba asume un sistema de ciencias de la educación que reconoce a la Pedagogía como ciencia y núcleo del resto, por ser ella la única que tiene como su único objeto de estudio la educación. Se reconocen como fundamentos de la Pedagogía a la Filosofía de la educación, la Sociología de la educación y la Psicología de la educación, de ahí la importancia de abordar y someter a la discusión en la actualidad, temáticas relacionadas con el surgimiento y conformación del sustento teórico de la Pedagogía.

En ese sentido, Chávez, J. Suárez, A y Permuy, L D. (2005) revelaron que "Los fundamentos teóricos de carácter sociológico de la pedagogía en Cuba se debilitaron, al desaparecer la sociología por varios años de los planes de estudio de las universidades y ser sustituida por el materialismo histórico". (p. 23).

Por ese motivo, se han editado algunas obras y ensayos sobre esa área del saber. Así, López, Miranda, Cobas, Valera y Chávez (2000), reconocen la existencia de posiciones propiamente sociológicas de la educación durante la República neocolonial (1902-1958) en educadores como Enrique José Varona, Roberto Agramonte, Laredano del Campo, Fanny Ascuy, Martín Rodríguez Vivanco, entre otros. Este último, fue un valioso exponente de ese período que contribuyó al desarrollo de la educación, de manera especial, al plasmar sus concepciones relacionadas con la sociología de la educación y la inspección escolar en obras que en la actualidad constituyen clásicos antecedentes para el estudio de esa materia.

En el caso de López et al. (2000) solo se hace referencia al objeto de estudio de la sociología pedagógica expuesto por Martín Rodríguez Vivanco en su texto Introducción a la sociología pedagógica de 1937.

En este estudio, se parte de considerar a la sociología pedagógica una ciencia y antecedente de la sociología de la educación.

Autores como Blanco (2004), consideran que la sociología pedagógica, también llamada sociología educativa o educacional, constituye un tipo particular de sociología de la educación que se desarrolló en las primeras décadas del siglo XX con notable éxito entre los pedagogos y algunos aportes inobjetables para el desarrollo posterior de los estudios sociológicos de la educación. 
Este autor ha reconocido, que el libro Introducción a la Sociología Pedagógica de Martín Rodríguez Vivanco de 1937, fue el primero publicado en español y dedicado a la sociología de la educación, por lo que debe considerarse un clásico en ese campo, aunque, no empleó sus concepciones como antecedentes para la conformación de su libro.

Por otra parte, para Rivero y Proveyer (2005), la sociología pedagógica es uno de los primeros antecedentes de la sociología de la educación, “... el punto inicial de conformación de la sociología de la educación". (p. 4) El libro es fundamentalmente una compilación de artículos de autores extranjeros que se relacionan directamente con el contenido de la asignatura Sociología y Política Social de la Educación que se imparte en la carrera de Sociología en la Universidad de La Habana.

De manera general, en la literatura pedagógica actual no se refleja la contribución de Martín Rodríguez Vivanco a la sociología de la educación en Cuba. Se aprecia desconocimiento de sus concepciones teóricas acerca de la familia, la comunidad, el objeto de estudio de la sociología pedagógica, los términos individualización y socialización del sujeto, la diferencia entre la sociología pedagógica y de la educación, entre otros que pueden considerarse el punto de partida para el estudio de esta ciencia en el país.

Por todo lo expresado anteriormente, el presente trabajo persigue como objetivo revelar la contribución de Martín Rodríguez Vivanco a la Sociología de la educación en Cuba durante la República neocolonial y la trascendencia de sus concepciones hasta la actualidad.

\section{Desarrollo}

La educación cubana tiene una deuda histórica con el pedagogo Martín Rodríguez Vivanco, que durante la República neocolonial se desempeñó como un valioso exponente del sector. Supo estar a la altura de su tiempo y dejar obras escritas que constituyen clásicos de obligada consulta en el país para investigadores de la inspección escolar y la sociología de la educación.

Martín Rodríguez Vivanco (19001985) fue un maestro cubano que nació en Quiebra Hacha, poblado rural del municipio Mariel, perteneciente entonces a la provincia de Pinar del Río. Realizó sus primeros estudios en una escuela organizada por los bautistas de su localidad y en lo adelante estaría vinculado a las labores de la Iglesia, donde llegó a ocupar el cargo de Presidente de las Escuelas 
Bautistas de Cuba Occidental, por más de 15 años consecutivos.

Emigró a la capital para formarse como maestro y desde allí, supo dar respuesta a necesidades educacionales que se presentaron en su contexto de manera creativa, lo que fue posible, debido al alto nivel de preparación docente y científicoinvestigativo, al vasto conocimiento de las disciplinas pedagógicas, el pasado educativo del país, así como al excelente dominio que poseía del idioma inglés y la capacidad de dirección.

Este pedagogo puso sus potencialidades al servicio de la educación nacional, produjo importantes textos docentes, ocupó responsabilidades llegando a desempeñarse como decano de la Escuela de Pedagogía de la Universidad de La Habana, participó en reformas educativas, introdujo asignaturas en los planes de estudio, elaboró proyectos, programas y libros de texto e impartió la docencia en instituciones públicas y privadas.

La Sociología de la educación como ciencia fue uno de los temas más recurrentes en la obra escrita de Martín Rodríguez Vivanco, de ahí, la necesidad de profundizar acerca del devenir histórico de la misma y poder apreciar la significación de sus concepciones para la educación cubana en aquel período y su trascendencia.

La revisión bibliográfica realizada reveló que entre las principales obras nacionales que abordan contenidos relacionados con la Sociología de la educación se destacan Rodríguez (1937, 1943, 1953), en el siglo XX, y más recientemente López et al. (2000), Blanco (2004), Rivero y Proveyer (2005).

El desarrollo de este artículo se estructura en tres epígrafes que inician con las consideraciones teóricas que sustentan el estudio de esta temática, le sigue una breve referencia al desarrollo del tratamiento sociológico de la educación en Cuba hasta inicios de los años treinta del siglo XX y culmina con las concepciones teóricas de Martín Rodríguez Vivanco sobre la Sociología de la educación.

\section{Consideraciones teóricas que sustentan el estudio de esta temática}

Para dar cumplimiento al objetivo propuesto, es preciso esclarecer primeramente el vocablo contribución. Este término es muy recurrente en investigaciones de todo tipo. Las de corte histórico, con frecuencia ahondan en su definición. Se asume la propuesta de Buenavilla (2004), como resultado de la sistematización resultante del proyecto Figuras destacadas de la cultura nacional: 
contribución al desarrollo de la educación y la teoría pedagógica cubana, a partir de las conceptualizaciones presentadas por Pérez, F, Álvarez, Z, Barrabia, O, Ortega, D y Piclin, J en sus tesis de doctorado.

Se asume la definición de Buenavilla (2004), que luego de sistematizar los criterios de los autores anteriores, arribó a la conclusión de que se puede hablar de contribución a partir de:

- Entender las potencialidades que presenta el educador como aquellas capacidades inmanentes a la naturaleza de su personalidad $\mathrm{y}$ obra que generan o constituyen fuentes en disposición de tributar al campo teórico, práctico o metodológico; capacidades que son portadoras de valores cognitivos $\mathrm{y}$ educativos, éticos y estéticos; posibilidad de ejercer influencia educativa en los demás.

- Esta contribución está estrechamente vinculada a las necesidades de desarrollo propias del fenómeno, y/o a las demandas que la sociedad le está planteando a la educación o al campo teórico en un momento histórico determinado.

- La interrelación dialéctica entre el educador y otras personas, y/o la educación, y/o campo teórico, que puede ser simultánea, continua o mediada por el tiempo y las instituciones y organizaciones sociales.

- Los resultados. (p. 5)

La definición anterior permite reconocer las potencialidades de Martín Rodríguez Vivanco, entre las que se destaca su comportamiento ético y moral apropiado que le permitieron ejercer influencia educativa en los demás, de manera directa con su actuación profesional y ciudadana e indirecta por medio de la producción de obras de variado formato.

Otros elementos que le permitieron contribuir al desarrollo de la educación, están asociados con el estrecho vínculo del educador con personalidades e instituciones dentro y fuera del país que le permitieron sostener intercambio teórico y práctico. Como resultado contribuyó al desarrollo de la sociología de la educación, a partir de sus concepciones acerca de esta ciencia, que serán abordadas más adelante.

Breve referencia al tratamiento sociológico de la educación en Cuba hasta inicios de los años treinta del siglo XX

Para comprender la contribución de Martín Rodríguez Vivanco al desarrollo de sociología de la educación, es preciso primeramente conocer el estado de esta 
ciencia en Cuba, antes de que este profesor redactara sus obras relacionadas con esta materia.

El tratamiento sociológico de la educación en el país inicia en el período Colonial, cuando intelectuales como José Agustín Caballero, Félix Varela, José de La Luz y Caballero, José Antonio Saco y José Martí Pérez, influenciados por la filosofía ilustrada europea, el empirismo y el sensualismo característico de la modernidad se orientan hacia la tendencia filosófico-sociológica de esta área del conocimiento. Todos ellos se pronunciaron en su momento en contra del escolasticismo y se adelantaron al surgimiento de la sociología general y de la sociología de la educación específicamente. Sus reflexiones acerca del fenómeno educativo han sido reflejadas desde un enfoque filosófico social.

Múltiples son las expresiones sobre la sociología de la educación que aparecen plasmadas en sus escritos. En ellos se aprecia como núcleo teórico de su pensamiento la educación popular. El padre Varela (1829), abogó por la preparación intelectual y una sólida formación ideológica de la juventud. Al respecto señaló: "El fomento de la instrucción pública es una obligación que puede llamarse popular. La necesidad de instruir a un pueblo es como darle de comer, que no admite demora". (p. 315)

Este autor reconoció, además que en la sociedad todos educan y el efecto de ese proceso es diferente, como desigual es la relación entre los agentes que intervienen en él, por ejemplo, señaló: “... un padre, un pariente es un maestro continuo a quien se le tiene mucha consideración y afecto". (Varela, 1992, p. 140).

José de La Luz y Caballero (18001862), realizaba el análisis en relación con la educación y la sociedad desde un enfoque pedagógico-sociológico. $\mathrm{La}$ sociología de la educación lucista es apreciable en su quehacer práctico, por ejemplo, cuando realizó de manera pública un ensayo del método explicativo con un éxito asombroso y así acercaba la escuela a la comunidad.

Otra idea progresista de este educador fue su propuesta de “... crear asilos o casas de corrección para adolescentes, escuelas en cuarteles y fortalezas, como en cárceles y presidios". (Rodríguez, 1926, p. 37) De igual modo abogaba por el acercamiento de la escuela a los hogares y gestionó la creación de una escuela para sordomudos, lo que viene a ser un verdadero reclamo de educación para todos.

Por su parte, el Apóstol de la independencia de Cuba, José Martí Pérez, 
le confirió a la educación una profunda dimensión político social que lo llevó a afirmar: "Una ciudad es culpable mientras no es toda ella una escuela". (Martí, 1890, p. 414-415). Evidentemente, existía claridad en sus ideas acerca de la influencia educativa que ejerce la sociedad como un todo armónico sobre el individuo.

En Martí se desarrolló ampliamente el aspecto social de la educación independientemente del sexo, raza, condición social u otro factor. Concebía que "La escuela ambulante es la única que puede remediar la ignorancia campesina." "Urge abrir escuelas normales de maestros prácticos, para regarlos luego por valles, montes y rincones..." (Martí, 1884, p. 291). Esta reflexión expresa la relación educación-sociedad en la obra martiana, el profundo humanismo manifiesto en el compromiso social que deben contraer los hombres para con otros hombres.

Por otra parte, expresó: “... todo esfuerzo por difundir la instrucción es vano, cuando no se acomoda la enseñanza a las necesidades, naturaleza y porvenir del que la recibe". (Martí, 1885, p. 327). Las palabras del apóstol refuerzan la idea de que cuanto más vinculada esté la preparación que reciba el estudiante al contexto comunitario, más comprometido estará con él.
Otro pedagogo de ideas muy avanzadas acerca de la educación durante la colonia fue Manuel Valdés Rodríguez. En relación con la necesaria articulación que debe existir entre la escuela y el medio social, sus agencias y agentes para garantizar su labor, expresó. Citado en Escalona (1959):

La necesidad suprema con todo, es armonizar el medio en que se educa. Querer educar al hombre divorciándolo de las influencias de su tiempo y de su sociedad, es pretender un absurdo. Los cargos que algunos formulan contra la escuela nacen propiamente de la inocente opinión de que ella, por si sola y única, es la panacea de los males de la humanidad... Señores, no es necesario repetirlo, cortar la corriente de solidaridad y simpatía entre la escuela y los factores sociales, equivale a convertir aquella en una especie de mecanismo arbitrario, artificial y armónico, propio solo para romper la ley de la unidad... ¿Comprometen esos factores el problema y la obra de la escuela? No cabe duda alguna en afirmarlo... (p. 122).

Los padres fundadores de la pedagogía cubana reconocían el estrecho vínculo de la educación con las esferas macrosociales (economía, política, ideología, cultura), especialmente con la independencia nacional y la abolición de la esclavitud.

En la década de los años 60 y en el transcurso de la propia guerra de los Diez años (1868-1878), los sectores de la clase 
media se interesaron por extender los servicios educacionales a la incipiente clase obrera. Durante la guerra se alfabetizó y educó a los campesinos y esclavos libres. La educación avanzaba más en la práctica que en la teoría.

En sentido general, López et al. (2000) aprecia la presencia de rasgos comunes en las ideas de estas personalidades en el período colonial como:

- Reconocimiento de la necesidad de la educación para el progreso social y el mejoramiento y bienestar de los pueblos

- Elevación del nivel cultural y educacional del pueblo como criterio esencial para alcanzar y valorar el progreso de un país

- La función social de la educación dirigida no sólo a la acumulación de conocimientos sino a la formación y el desarrollo del hombre para la vida

- El carácter democrático de la educación al extender el servicio educativo a los diferentes sectores y capas sociales

- Crítica a los males y factores sociales que afectan la educación

- La necesidad de vincular el ideal de educación a las necesidades concretas de la isla
- Análisis de las consecuencias sociales negativas, que provocan la utilización del método escolástico en la organización del contenido de la enseñanza para la formación de la personalidad". (p. 55).

Por su parte Varona (1992), reflexionaba acerca de la necesidad de elaborar una teoría sociológica educacional, que reflejara los problemas educativos de la Isla. En 1899 planteó claramente que:

... en la sociedad todo educa y todos educamos... Pero aún nos faltará mucho para llegar a ese fin, mientras la colaboración de la familia y la sociedad, en general, en la obra de convertir a los niños en hombres no sea aferrada y no se conspire en la misma dirección que la disciplina escolar... (p. 7).

Las ideas anteriores están en función de la realidad concreta, establecen una relación teoría-práctica, expresada en el vínculo de los intereses de los estudiantes con los de la comunidad en que viven.

Algunos investigadores cubanos entre ellos López et al. (2000), ubican el proceso de institucionalización de la Sociología de la educación en Cuba en el período comprendido de 1930-1933, como resultado del esfuerzo personal de prestigiosos intelectuales que fundaron cátedras de sociología en las escuelas normales, los institutos de segunda 
enseñanza y la Universidad de La Habana, entre los que destacan a Enrique José Varona, Martín Rodríguez Vivanco, Roberto Agramonte, Laredano del Campo y Fanny Ascuy.

Las consideraciones acerca de la sociología de la educación de los pedagogos de la Colonia y las primeras décadas de la República aparecen recogidas en documentos de diverso formato como epistolarios, discursos, manuscritos, en ocasiones incluidos en documentos de contenido muy general. En esas condiciones resulta difícil conformar un texto docente, sin embargo, no se aprecia rompimiento entre las concepciones de los precursores de la pedagogía cubana y Martín Rodríguez Vivanco, que fue un ferviente seguidor del legado pedagógico del siglo XIX.

De ahí, la importancia de exponer la contribución de Martín Rodríguez Vivanco a la Sociología de la educación en un período tan complejo de la historia de Cuba como la República neocolonial.

\section{Concepciones teóricas de Martín Rodríguez Vivanco acerca de la Sociología de la educación}

El epígrafe se sustenta en la periodización realizada por la propia autora Mesa (2013), sobre la obra educativa de Martín Rodríguez Vivanco, que estableció como criterio el impacto de su producción intelectual en el desarrollo de la educación cubana.

La producción intelectual, a criterio de Añorga (2012) “... es la producción inmaterial de los hombres, abarca todas las actividades humanas, donde se manifieste la creación, aunque se reduzca a una nueva forma de exponer los conocimientos y resultados de otros hombres". (p. 8).

Añorga (2012) clasifica la producción intelectual en científica, técnica y humanista y las define así:

La producción científica, está relacionada con la producción de conocimientos, se expresa mediante investigaciones, sistematizaciones, producción de conocimientos, teorías, ciencia consolidada. La producción técnica: (solución a problemas prácticos concretos, inventos, diseños, modelos, adaptaciones, planes y proyectos). $\mathrm{La}$ producción humanista: (creación y difusión de valores) filosofía, literatura, artes plásticas, música, cultura, educación y deportes. (p. 8).

Mesa (2013) en su estudio determinó las siguientes etapas para el periodizar la obra educativa de Martín Rodríguez Vivanco:

Etapa I. Surgimiento de su obra educativa (1919-1926)

Etapa II. Impulso productivo de su obra educativa (1927-1938) 
Etapa III. Perfeccionamiento y consolidación de su obra educativa (19391953). (p. 57).

Desde la primera etapa en que comienza a formarse en la Escuela Normal para Maestros de La Habana recibe la influencia de educadores insignes de la nación entre los que se destacan Ramiro Guerra y Arturo Montori. El acercamiento a estas figuras lo inclina hacia el estudio de las raíces educacionales cubanas.

Las concepciones expuestas en este epígrafe, corresponden a las tres etapas, en las cuales este autor produjo algunas obras relacionadas con la sociología de la educación, que como parte de su producción intelectual, sirvieron también para delimitar las etapas. Estos textos permiten apreciar la madurez de sus concepciones acerca de la sociología de la educación de una etapa a la otra.

Desde el año 1926 en que publicó el artículo El problema campesino, dejó claro que a pesar de haber emigrado hacia la capital y permanecer en ella, estaba comprometido con su origen de clase humilde y sus raíces rurales.

En el artículo, más que revelar las penurias que padecía el campesinado demandó transformaciones generales para su situación. Así, aunque desde una posición idealista, exigía públicamente al gobierno que diera el ejemplo y entregara tierras a los campesinos, que hasta el momento no eran dueños ni de sus propios brazos.

Este pedagogo tuvo la valentía social de declarar el problema existente y enfatizar en el papel del estado, como principal causante de los males del campesinado en Cuba. Años más tarde, por la importancia que le confería al asunto, incluyó el artículo en su libro Introducción a la Sociología Pedagógica en el capítulo Comunidad rural, contenido que pasó a formar parte del legado pedagógico y social de este autor, donde se evidencia la maduración de sus ideas y la toma de partido por los humildes.

La reflexión anterior expresa cómo él luchó por reformas sociales y extender los servicios educacionales al sector rural y para ello se valió fundamentalmente de la docencia y los medios de prensa.

Martín Rodríguez Vivanco desde 1934, año en que se crea la Cátedra de Sociología pedagógica y Técnica de Inspección Escolar en la Escuela de Pedagogía de la Universidad de La Habana, estuvo a cargo de la conformación de los planes de estudio y programas de ambas asignaturas e incluso de la elaboración de los libros de texto. Rodríguez (1943) sostenía el criterio de que: 
... la transformación incesante de la sociedad actual puede también ser considerada la causa que determina ese carácter cambiante, variable, de los planes de estudio. De lo contrario estaríamos expuestos a ofrecer una educación inadecuada completamente atrasada, a la nueva generación a nuestro cargo. (p. 26).

Lo anterior explica las adecuaciones a planes, programas y libros de texto que realizara con frecuencia.

En 1937, escribió el primer libro de texto y lo tituló, Introducción a la sociología pedagógica. El material contenía las explicaciones de cátedra del autor con el empleo de los conocimientos más actualizados a nivel mundial sobre la temática, los que debió traducir del inglés. Con profundo respeto expuso los contenidos auxiliándose de las consideraciones teóricas de relevantes personalidades en el estudio de esta materia como Durkheim E, Comte A y en mayor número citó autores norteamericanos, con muchos de los cuales sostuvo intercambio académico.

Martín Rodríguez Vivanco se mantuvo a la altura de su época, de ahí que asumiese los adelantos didácticos del movimiento de la Escuela Nueva o Activa y los adecuara creadoramente al contenido de sus materias.

Esta tendencia pedagógica ha sido objeto de estudio de importantes personalidades de la educación. En el ámbito internacional puede citarse a Ferriére. (1922), Luzuriaga. (1960), Octavi. (1978), Zubiria. (1995), entre otros. En el caso de Cuba ha sido estudiada por Sánchez-Toledo. (s/f), Chávez. (s/f), Valera. (2003) y un grupo de investigadores del Centro de Estudios para el Perfeccionamiento de la Educación Superior (CEPES), entre otros.

El activismo es un movimiento de escuelas nuevas caracterizado por la aplicación de la psicología a la pedagogía sin preocupaciones teleológicas. Se caracterizada por tres aspectos fundamentales:

- La concepción de la infancia y su repercusión en la educación

- La concepción del profesor y su papel en la educación

- La renovación metodológica.

En sentido general, este movimiento centra su atención en el alumno, potencia su activismo. El profesor es un guía para la orientación de la experiencia del alumno, debe estimular la actividad con el empleo de métodos renovadores, entre los que se destaca la atención a las diferencias individuales, la motivación, la socialización y la cooperación escuelafamilia, entre otros. Implicaba profunda transformación de la institución docente, la familia, la sociedad, elevada preparación 
de los educadores para asumir los altos retos de la enseñanza y la participación protagónica de los alumnos, que rompería con los esquemas tradicionales. Significó progreso con relación a las concepciones y prácticas de los siglos anteriores.

Los avances en la educación cubana se recogen básicamente en las principales obras publicadas en la etapa, las cuales se hicieron eco de los adelantos educativos de Cuba y el mundo. Merecen referencia los textos: Didáctica de la Escuela Nueva, de Aguayo A M, Didáctica y dirección del aprendizaje de González D, Introducción a la Sociología Pedagógica y Técnica de la inspección escolar, de Martín Rodríguez Vivanco, entre otras.

Los pensamientos expuestos en las obras citadas, en su mayoría, no encontraron aplicación práctica, aunque fueron socializados también a través de revistas pedagógicas, libros de texto, periódicos y la docencia como parte de los contenidos de las asignaturas impartidas en las escuelas normales y en la carrera de Pedagogía en la Universidad de La Habana, de modo que sus avances novedosos pasaron a formar parte del modo de actuar de muchos educadores.

En Cuba, los seguidores de la Escuela Nueva de manera general tomaron sus adelantos didácticos y los adecuaron al contexto nacional. Se aprecia en ello como elemento novedoso la indiscutible huella del legado patriótico y humanista de las generaciones que antecedieron a estos educadores, quienes incorporaron como resultado de la evolución histórica de la tendencia el componente axiológico a los aspectos asumidos, lo que distinguió al movimiento en el país de sus manifestaciones en el resto del mundo.

Hay que considerar también que Martín Rodríguez Vivanco recibió la influencia directa de esta tendencia en Estados Unidos, país que visitó en reiteradas ocasiones en calidad de líder bautista en el cargo de Presidente de las Escuelas Dominicales de Cuba Occidental, como estudiante de posgrado o de manera intencional para sostener intercambio académico.

Estudió y analizó los postulados de ese movimiento de manera crítica, no los repitió miméticamente. No asumió el pragmatismo norteamericano que servía de base a esta tendencia. En la aplicación que hizo de la Escuela Nueva, se aprecia originalidad, acercamiento a las características e idiosincrasia del cubano y la consideración de la situación histórica. Sostenía que el fin de la educación es la formación del hombre que aporte a la sociedad y en ese sentido, expresó: "No se educa al individuo para sí mismo. La sociedad asume esa función por él a fin de 
que sea educado para la sociedad antes que para sí mismo”. (Rodríguez, 1943, p. 23).

En las obras que escribió este pedagogo sobre sociología de la educación, trató acerca del objeto de estudio de la sociología pedagógica, su relación con otras ciencias, las categorías socialización e individualización del sujeto, las agencias de socialización educativa, el papel del maestro, la familia y la comunidad, entre otros tópicos.

En el libro Introducción a la Sociología pedagógica no ofreció una definición personal del término sociología pedagógica, lo que no debe considerarse una limitación, si se tiene en cuenta el estado incipiente de esta ciencia en aquel momento. En su lugar, asumió a David Snedden, que la considera "el conjunto de conocimientos científicos que derivándose de la sociología general pueden ser usados en la práctica educacional, así como en la dirección del proceso en ese campo de trabajo". (p. 27).

Esta obra constituye un valioso testimonio que prueba que Martín Rodríguez Vivanco consideró la Sociología Pedagógica o Educacional una ciencia aplicada cuyo objetivo fundamental “... es la determinación de los fines $\mathrm{y}$ objetivos de la educación” (Rodríguez, 1943, p. 26), lo que viene a ser un planteamiento de mucha generalidad, aunque llegó a reconocer que esta ciencia no solo analiza las deficiencias y las fortalezas de un agente determinado, debe sugerir soluciones y procedimientos prácticos para su mejoramiento. Consideró a la sociología y la psicología pedagógicas como ciencias básicas de la educación y a ambas entre sí complementarias. Al respecto Rodríguez (1943) señaló:

La sociología pedagógica se encuentra en el grupo, junto a la psicología pedagógica, formando con ella los dos grandes pilares que sostienen el edificio de la educación. (p. 17). La educación como un campo de ciencias aplicadas completamente desarrollado, ha de emplear numerosas ciencias. $\mathrm{La}$ psicología le ayudará con dos clases de contribuciones importantes: a) la educabilidad de un estudiante dado o de tipos dados de aprendizaje; y b), cuáles son los mejores métodos para dirigir el proceso de aprendizaje. ( $\mathrm{p}$. 28).

Establece contactos entre la psicología y la sociología pedagógicas. La primera, “... parte del individuo y estudia el proceso de aprendizaje. La sociología pedagógica estudia las tendencias, capacidades, deseos y necesidades del grupo social.” (Rodríguez, 1943, p. 28).

La psicología y la sociología son pues complementarias; ambos estudios son necesarios para interpretar todas las posibilidades así del individuo como del grupo social. Como que la educación depende 
igualmente del proceso de aprendizaje y del proceso de socialización sus bases $\mathrm{o}$ fundamentos descansan sobre la psicología pedagógica y la sociología pedagógica (p. 28).

El autor define los términos educación y escuela, que resultan indispensables cuando se habla de sociología de la educación.

La categoría educación la trató en sentido general, en toda la magnitud que la misma encierra, pues comprendió la influencia que ejerce la sociedad como un todo sobre el individuo, lo que se expresa en el reconocimiento que hace de las agencias educativas. Sin embargo, Rodríguez (1943) logra distinguir la educación escolarizada cuando, señala que:

.. la educación adquiere caracteres de mayor organización $\mathrm{y}$ notamos que se encuentra a cargo de personas especialmente dedicadas a ese trabajo, constituyendo una acción sistemática ... para la transmisión del saber, que se lleva a cabo constantemente y según un plan de estudios y en un curso de cierta duración ... una educación prolongada. (p. 308).

Con esta reflexión reconoció el carácter organizativo, continuo y constante de la educación.

El autor considera que la escuela “... es la principal agencia educativa puesto que tiene como función esencialísima encargarse de la educación". (Rodríguez, 1943, p. 300).

Según su criterio, "Ninguna otra institución necesitó tanto el tratamiento sociológico como la escuela, creada precisamente por la sociedad como medio para dar al individuo la indispensable preparación para que ocupe dignamente su puesto en el agregado social". (Rodríguez, 1943, p. 14). "La escuela, ha sido organizada como una institución diferenciada y especializada que tiene a su cargo la tarea de iniciar al joven en los más recónditos aspectos de la vida cultural". (p. 74)

El pedagogo compartió en el claustro universitario con educadores que se pronunciaron en contra de la coeducación mientras él se mostraba defensor de la misma. En ese sentido, se atrevió a afirmar que "Las escuelas que tienen especial interés en clasificar los niños según el sexo están actuando contra la naturaleza desde el punto de vista social...”. (Rodríguez, 1943, p. 227). Negó que la presencia del varón contribuya a masculinizar a la niña, ni la femenina afecte la orientación sexual del varón. "Cada uno acentúa las cualidades de su sexo en presencia del otro y aprenden a respetarse mutuamente y a tratarse con la debida corrección, además 
de conocerse mejor para futuras relaciones sociales". (p. 227).

Rodríguez (1943) señaló además que:

La escuela ensancha la comunidad. Es necesario estudiar y coordinar la naturaleza del niño y las acciones sociales de la sociedad. Las actividades de la comunidad a través de programas de investigaciones, excursiones, higiene, entre otros. La escuela como centro abierto a la comunidad. (p. 90).

De esa manera, le confiaba a la escuela el carácter de centro promotor de la cultura. Asumió la escuela como coordinadora de todas las agencias de socialización educativa, la cual se debía convertir en su centro.

Martín Rodríguez Vivanco refiriéndose a la comunidad consideraba hacer extensivo el activismo de los alumnos a la comunidad, cuando se planteó ocupar el tiempo de la niñez y la juventud en actividades formativas. Insistió en el aprovechamiento necesario del edificio escolar en horarios extraescolares por la comunidad.

Un antecedente de la sociología de la educación se aprecia en la referencia que hizo de las agencias de socialización educativa cuando citó a la escuela, la familia, las iglesias, asociaciones diversas, clubes, corporaciones de distintos tipos, los campamentos de verano, el museo escolar, la biblioteca, el teatro, la prensa, la radio, el cinematógrafo, entre otras que “... ofrecen grandes oportunidades para la adquisición de la cultura". (Rodríguez, 1943, p. 144).

La sociedad cubana actualmente está enfrascada en lograr el acercamiento necesario entre el hogar y la escuela. Martín Rodríguez Vivanco al referirse a la cooperación escuela -hogar con énfasis en la asistencia a clases, la ayuda con las tareas, reconocía que la escuela como aliada del hogar está obligada a mantener con él una constante cooperación, necesita de la ayuda que los padres pueden prestar para que su trabajo sea eficaz. "La escuela debe penetrar en el medio vivido y estudiar las condiciones existentes en la comunidad, llegar hasta el hogar y comprender los problemas que afectan al educador para acudir eficazmente en su ayuda”. (Rodríguez, 1943, p. 287).

Este educador sostenía que la escuela debe compensar la decadencia de la acción educativa del hogar, debía garantizar: “... el desarrollo de la habilidad física, de la habilidad intelectual, la preparación vocacional, la cooperación entre el hogar y la escuela..." (Rodríguez, 1943, p. 69).

En ese sentido, se refirió a establecer mecanismos de cooperación escuela hogar, a partir de la creación de 
organizaciones de padres y maestros, clubs de madres, sociedades de amigos de la escuela. Nada más cercano en la actualidad al Consejo de escuela y a los padrinos de los centros educacionales, debido a la necesidad de crear también alianzas estratégicas.

La familia a criterio de este pedagogo “... es el más importante de los grupos que los sociólogos llaman primarios y se distinguen porque sus miembros mantienen contacto personal, cara a cara". (Rodríguez, 1943, p. 83).

Agrega más adelante en esta obra, que:

Desde el punto de vista sociológico la razón suprema de la existencia de la familia es su demostrada eficacia como institución más adecuada para la crianza del niño y la transmisión al mismo de la mayor parte de la herencia social. (p. 83).

Martín Rodríguez Vivanco al igual que los educadores que le antecedieron abogó por la educación popular, sin distinción alguna. En ese sentido, señaló: "Un buen sistema de educación urbana debe inscribir toda su población escolar y brindarle la oportunidad de adecuado aprendizaje”. (Rodríguez, 1943, p. 155).

Es meritorio destacar, que este autor reconoció la estrecha relación individuosociedad, expresada mediante la categorías fundamentales cuando se habla de sociología de la educación. Sin embargo, en psicología por ejemplo resulta común utilizar el concepto de personalidad para referirse a individualidad. Rodríguez (1943) reconocía que, en este sentido, es importante partir de:

¿Qué cosa es la personalidad y cómo se forma? ¿De qué manera se encuentra constituida? Nos encontramos frente a un asunto difícil de explicar, acerca del cual los psicólogos y pensadores no han realizado todavía investigaciones suficientes, problema acerca del cual estamos lejos de poder decir la última palabra. (p. 247).

En la actualidad no existe un consenso general sobre la definición del término personalidad, diferentes ciencias, como la psicología, la pedagogía, la psiquiatría, la sociología, antropología, criminología, entre otras, incluyen esta categoría en sus estudios.

Rodríguez (1943) consideraba “... la personalidad como síntesis de las tendencias, como resumen de los impulsos interiores y de las adquisiciones del medio circundante, el medio físico y social en que vivimos". (247).

Como se aprecia en las consideraciones de este autor, un papel importante en la formación de la personalidad recae en el contexto, el medio socialización y la individualización, dos 
social, en la asimilación de los contenidos sociales.

En su momento al referirse a los factores determinantes de la personalidad consideraba "que el temperamento sea básico, interviniendo la inteligencia, el carácter y las otras adquisiciones durante la vida del individuo, como son los hábitos, los conocimientos, las aptitudes, etc." (Rodríguez, 1943, p. 247).

A criterio de este pedagogo:

En un medio social cualquiera el individuo está sometido a la presión de la tradición, los hábitos de conducta, los sentimientos, la cultura, etc., de cuantos le rodean; pero no se somete de forma pasiva a tales influencias. Reacciona y llega a trasmitir sus sentimientos y experiencias propias, realizándose un verdadero intercambio, produciéndose el equilibrio al formarse la personalidad. (p. 253).

Como se puede apreciar le atribuye un papel activo, protagónico al sujeto en formación, lo que se corresponde plenamente con los presupuestos de la Escuela Nueva.

A su juicio:

... la individualización equivale a la formación de la personalidad, por el desarrollo de todos aquellos elementos que contribuyen a la edificación del carácter; y la socialización, se refiere a la adaptación o ajuste del individuo a la manera de vivir y trabajar del grupo a que va a pertenecer. (p. 245).
Agregó, además, refiriéndose a la individualización que:

Estará mejor preparado para las funciones sociales, para la defensa del grupo, para trabajar en pro de la comunidad, el que ha logrado el fortalecimiento de su individualismo, de la parte personal o egoísta de la vida. Lo necesario será encausarlo hasta altos ideales y normas de acción social. (p. 247).

Es apreciable cómo se reflejan los principios que él sustentaba en lo personal, en sus concepciones educativas, cuando asume, por ejemplo, que el individuo debe seguir las normas de conducta establecidas por los grupos a que pertenece, apropiándose de los códigos sociales. Insiste en que se forma al individuo para la sociedad.

La comunidad como medio que rodea al estudiante $\mathrm{e}$ incide en su formación cultural, también fue objeto de análisis en sus obras. Insistió en las potencialidades educativas que ofrece la comunidad, al contar con una o más escuelas, diversas instalaciones, empresas, programas de higiene y salud.

Resulta válido destacar cómo este autor no olvida su origen rural. En este libro dedicó dos epígrafes a la comunidad rural, expuso sus tipos y características, con énfasis, en las condiciones ecológicas, que devienen en la actualidad en un eje 
transversal del sistema educacional cubano como es la educación ambiental. El autor enfatizó en las consecuencias que para la cultura y la educación se derivan de su estudio en la comunidad rural.

Martín Rodríguez Vivanco reconocía la preparación que debe tener el maestro para trabajar en la comunidad rural. Establecía como criterio que lo ideal es que proceda de ese medio, por su sensibilidad ante los problemas del campesinado. Por ello, insistía en el papel que debe jugar la educación en la transformación de la comunidad rural en general. Al respecto Rodríguez (1954) expresó:

Es indiscutible que en las condiciones que rodean esa comunidad social, que impiden el mayor bienestar y el mayor progreso de la misma, ha de desempeñar la educación un papel trascendente en la responsabilidad de variar las condiciones actuales para lograr otras que son las deseables y las que deben existir. (p. 178).

Al hablar de la historia de la educación cubana y en especial del pensamiento educativo de sus principales personalidades, es imprescindible abordar el tema de la moral, objeto de constante preocupación para estas figuras. En ese sentido, un elemento distintivo con relación a los postulados de la Escuela Nueva en las concepciones de Martín Rodríguez Vivanco, es el estrecho vínculo entre cognoscitivo y lo axiológico, expresado en el aprovechamiento que hacía de las potencialidades educativas del contenido de sus textos docentes, para así fomentar la formación de valores, convicciones e ideales en sus estudiantes a través de mensajes certeros.

Lo axiológico en Rodríguez (1943) se manifiesta firmemente cuando expresó:

La educación puede formar ideales dinámicos, capaces de mover a los grandes grupos sociales. Lo prueba la labor elevada que han tenido los educadores en casi todos los movimientos de renovación social y de progreso ... En nuestra historia nacional podemos referirnos al movimiento educativo de fines del siglo XIX, que precedió nuestra guerra de emancipación. (p. 287).

Sobre los valores sociales Rodríguez (1943) consideró que:

... estarán siempre en relación con la época, tendrán también relación con las aspiraciones de los individuos o los grupos sociales, pero no pueden ser definitivos ni permanentes. Han de estar siempre en constante renovación. Los valores sociales fijados para un momento, para un pueblo, pueden ser más tarde descartados por ese mismo pueblo o considerados como falsos por los demás. (p. 296).

Concebía así el papel cambiante que tienen en la sociedad los valores en cuanto al nivel de jerarquía. 
Llevó los valores al contenido de la asignatura Sociología Pedagógica, que le sirvió además para colectivizar los métodos de enseñanza grupal, los que consideró eran empleados para cultivar el pensamiento constructivo y los hábitos de concentración mental. Por ejemplo, planteaba que: "En el juego y en el trabajo escolar se introducen los más elevados ideales $y$ se hace responsables a los alumnos de su propia conducta...". (Rodríguez, 1943, p. 57). En otro momento subrayó: "El sentimiento patriótico, por ejemplo, puede constituir una poderosa fuerza que lleva a un individuo al heroísmo y reunido con otros de condiciones semejantes produce las gloriosas hazañas de que está llena la historia de todos los pueblos". (Rodríguez, 1943, p. 287).

La asunción por este pedagogo de los adelantos de la Escuela Nueva constituye un ejemplo claro de cómo se deben conocer, divulgar y justipreciar las tendencias pedagógicas y los adelantos científicos universales sin perder la esencia de la nación, sus raíces, lo autóctono y con ello contribuir a impulsar la ciencia pedagógica de su tiempo.

Otro elemento que llega hasta hoy con una fuerza sorprendente es su criterio acerca del papel que desempeña el maestro como agente social en la sociedad. Al respecto, afirmó que “... la labor de la educación es una obra de buena voluntad y de entusiasmo enaltecedor". (Rodríguez, 1941, p. 289).

Este pedagogo compartió la docencia en centros públicos y privados, sin embargo, reconocía las ventajas de la educación pública. Esto se evidencia cuando señaló: "El trabajo de la escuela pública consistirá en destruir las diferencias entre los grupos que componen las sociedades heterogéneas...”. (Rodríguez, 1943, p. 17).

Con la inclusión de la asignatura Sociología Pedagógica en el currículo de las escuelas normales, realizó una revisión de los contenidos a abordar, lo que dio origen a un texto más actualizado en 1953 , con el que cierra la tercera etapa, al que tituló solamente Sociología Pedagógica. Con este material Martín Rodríguez Vivanco intentó contribuir a “... establecer los fundamentos sólidos ... a los aspectos científicos de la pedagogía”. (Rodríguez, 1954, p. V).

En este libro, dejó plasmadas construcciones teóricas de incalculable valor para el desarrollo de la pedagogía cubana y expuso valiosas consideraciones acerca de la sociología de la educación. Superó concepciones de la obra anterior, en que asumió por ejemplo la definición sociología pedagógica de otro autor. En esta ocasión, llegó a ofrecer una definición 
provisional orientadora, cuando planteó “... la sociología pedagógica estudia los hechos sociales desde el punto de vista de la educación, o en sus relaciones con la educación”. (Rodríguez, 1954, p. 7).

Más adelante declaró los límites entre la sociología general y la sociología pedagógica donde puntualizó que la primera “... estudia todos los hechos sociales" y la segunda “... se circunscribe al campo especializado de la educación profesional y científica”. (Rodríguez, 1954, p. 7). La aclaración final complementa la definición dada por él, ya que ubica el campo de esta ciencia en la pedagogía (la relaciona con la escuela), lo que viene a corroborar la correspondencia con los criterios actuales sobre este término en Cuba.

En esta obra hizo un nuevo acercamiento a la relación individuosociedad. En ese sentido, expresó: “... el hombre es sociable por naturaleza, lo que equivale a reconocer que la existencia en el aislamiento es completamente imposible" (Rodríguez, 1953, p. 19).

En el libro incluyó un epígrafe sobre relaciones de la sociología pedagógica con otras ciencias y trató acerca del lugar que ocupa esta entre las ciencias de la educación. Así dejó claro, que ese asunto que tanto atrae la atención de los investigadores de la educación en el presente ya era tratado por los educadores durante la República neocolonial.

Rodríguez (1953), reconoció la necesaria relación de sociología pedagógica con otras ciencias, por ejemplo “... la estadística, la historia, la antropología”. (p. 17).

Consideraba que:

.. la biología y la psicología han contribuido con sus valiosos resultados a esclarecer los estudios de la naturaleza del hombre... Desde el punto de vista psicológico el hombre es un ser inteligente dotado de un equipo mental susceptible de amplio desarrollo en condiciones de favorables... No solo posee una capacidad de organización superior a todas las demás especies animales sino que cuenta con las facultades específicas para atesorar experiencia, fabricar instrumentos y desarrollar el lenguaje... es capaz de desarrollar los medios que le permiten colocarse en el más alto grado de progreso cultural. Ese gran desarrollo de la técnica se debe a su condición social. (p. 20).

El autor reconoce así una vez más la educabilidad del hombre, su capacidad intelectual, creadora y organizativa, que unido a su condición social le permite vivir y desarrollarse plenamente.

Plantea que Rodríguez (1953):

...el hombre nace como individuo sin que posea la condición de persona, la cual irá adquiriendo lentamente a medida que realiza el proceso de maduración en contacto con los demás miembros de la colectividad... La individualidad es, por tanto, el 
resultado del desarrollo de las potencialidades hereditarias $\mathrm{o}$ congénitas bajo la influencia de factores externos. (p. 21).

El autor asevera que la personalidad se forma y qué importante papel ejerce la socialización en ese proceso.

Resulta significativa su afirmación de que la sociología pedagógica se relaciona también con otras "sociologías especiales... por ejemplo, la sociología rural, urbana, jurídica, médica" (Rodríguez, 1954, p. 18), entre otras, se basa en la utilización de datos en determinados aspectos del estudio de esta ciencia. Llegó a reconocer que "Entre las sociologías especiales, que se relacionan con la sociología pedagógica, debemos mencionar a la sociología de la educación, que algunos confunden lamentablemente con la sociología pedagógica, cuando son dos materias completamente distintas". ( $p$. 18).

Aun cuando la Sociología pedagógica y la Sociología de la educación no son ciencias totalmente diferentes, como asevera este autor, porque surgen de una misma ciencia madre, la sociología y se relacionan con el fenómeno educativo. En el caso de la primera estudia los problemas sociales de la educación, no desde la Sociología, sino de la Pedagogía y para la enseñanza. Se preocupa por mejorar la calidad de la educación escolarizada y comprender los problemas sociales que la afectan, por lo que profundiza en los factores familiares y comunitarios de la educación. En tanto, la Sociología de la educación al enfocar el fenómeno educativo desde perspectivas sociológicas, colabora a explicar y transformar las relaciones que se establecen en la esfera educacional desde el nivel micro hasta el macro en la sociedad.

El reconocimiento por este autor de ambas ciencias indica progreso en sus ideas, ya que, al distinguirlas, incita a la reflexión en torno a una problemática que tanto ha costado a la ciencia, en la actualidad y es uno de los elementos que viene a corroborar la importancia de buscar respuestas en su obra.

Las concepciones educativas de Martín Rodríguez Vivanco en sentido general, adquieren una significación trascendental, ya que permiten conocer cómo se comportaban las ciencias de la educación durante la República neocolonial, permite reflexionar acerca de las relaciones entre la pedagogía y sus fundamentos o entre ella y otras ciencias sociales.

\section{Consideraciones finales}


Martín Rodríguez Vivanco fue un pedagogo que se destacó como digno exponente de la educación en Cuba durante la República neocolonial. Contribuyó a la Sociología de la educación mediante la docencia, la redacción de textos, planes y programas de estudios.

Sus concepciones estuvieron influenciadas por la tendencia pedagógica más progresista de la época, la Escuela Nueva, que supo adecuar con creatividad al contexto nacional. Expuso sus consideraciones teóricas acerca del objeto de estudio de la sociología pedagógica, su relación con otras ciencias, las categorías socialización e individualización, el papel de las agencias de socialización educativa como la escuela, la familia, la comunidad, distinguió a la sociología pedagógica de la sociología de la educación, con lo que incitaba a la reflexión acerca de las relaciones entre la pedagogía y sus fundamentos o entre ella y otras ciencias sociales.

Las concepciones de este pedagogo pueden considerarse punto de partida para el estudio de esta materia en el país, de ahí, la importancia de buscar respuestas en su obra escrita que sirvan para sustentar las raíces de la pedagogía cubana.

\section{Referencias}

Añorga, J. (2012). La producción intelectual: proceso organizativo $y$ pedagógico. La Habana: Editorial Universitaria.

Blanco, A. (2004). Introducción a la Sociología de la educación. La Habana, Cuba: Editorial Pueblo y Educación.

Buenavilla, R. (2004). Ficha de resultado 2. Proyecto: Figuras destacadas de la cultura nacional: contribución al desarrollo de la educación y la teoría pedagógica cubana. La Habana: Instituto Superior Pedagógico Enrique José Varona.

Buenavilla, R. (2007). Influencias educativas: factores objetivos y subjetivos. Dialéctica de su desarrollo. La Habana: Instituto Superior Pedagógico Enrique José Varona.

Comisión Nacional Cubana De La Unesco. (1992). Enrique José Varona. Trabajos sobre educación y enseñanza. La Habana: Editorial Pueblo y Educación.

Chávez, J. Suárez, A \& Permuy, L D. (2005). Acercamiento necesario a la pedagogía general. Editorial Pueblo y Educación.

Fullat, O. (1995). Filosofías de la educación. Barcelona.

López, J., Miranda, O L., Cobas, M., Valera, O., \& Chávez., J. (2000). Fundamentos de la Educación. La Habana: Editorial Pueblo y Educación.

Luzuriaga, L. (1960). La pedagogía contemporánea. Buenos Aires: Editorial Losada. S A.

Martí, J. (1884, mayo). Maestros ambulantes. La América. Nueva York. Obras Completas. Tomo. 8. 
Martí, J. (1885, 4 de diciembre). Los indios en los Estados Unidos. La Nación. Buenos Aires. Obras Completas. Tomo. 10.

Martí, J. (1890, marzo). Haití y los Estados Unidos. Periódico El Partido Liberal. México. Obras Completas. Tomo. 12.

Mesa, L. (2013). La obra educativa de Martín Rodríguez Vivanco (Tesis de doctorado). Universidad de Ciencias Pedagógicas Enrique José Varona, La Habana.

Rivero, Y., \& Proveyer, C. (2005). Selección de lecturas de Sociología y Política Social de la Educación. La Habana: Editorial Félix Varela.

Rodríguez, M. (1926). La labor pedagógica de la Sociedad Económica de Amigos del País (Tesis). La Habana: Universidad de La Habana.

Rodríguez, M. (1926). El problema campesino. El País. 17(1), 5-6.

Rodríguez, M. (1937). Introducción a la Sociología Pedagógica. La Habana: Editorial Cultural S A.

Rodríguez, M. (1943). Introducción a la Sociología Pedagógica. La Habana: Editorial Cultural S A.

Rodríguez, M. (1953). Sociología Pedagógica. I Parte. La Habana: Publicaciones Cultural SA.

Varela, F. (1829). El mensajero semanal. T. 1. 43. Nueva York.

Varela, F. (1992). Misceláneas filosóficas. La Habana: Editorial Pueblo y Educación.
Revista Brasileira de Educação do Campo y no participó en el proceso de evaluación del artículo.

Recibido en: $26 / 06 / 2017$ Aprobado en: 20/08/2017 Publicado en: 15/12/2017

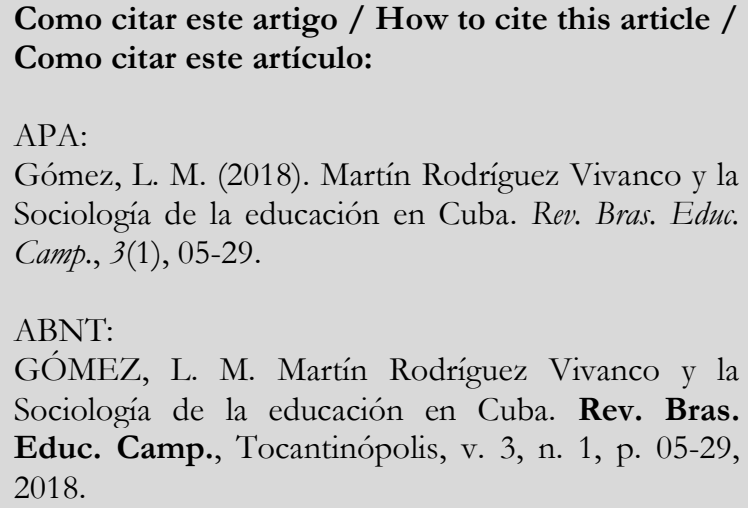
Sociología de la educación en Cuba. Rev. Bras. Educ. Camp., Tocantinópolis, v. 3, n. 1, p. 05-29, 2018.

\section{ORCID}

Lídice Mesa Gómez

http://orcid.org/0000-0002-2121-5161

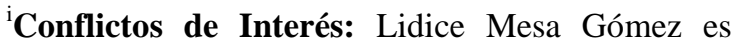
miembro del Consejo Editorial Internacional de la 